\title{
New Version for Hardy and Rogers Type Mapping in Dislocated Metric Space
}

\author{
P Sumati Kumari ${ }^{*}$, V Vasantha Kumar ${ }^{1}$, I Rambhadra sarma ${ }^{2}$ \\ * Research Scholar \& Assistant Professor in K L University . \\ Email: mumy143143143@gmail.com \\ ${ }^{1}$ Department of Mathematics, K L University, Vaddeswaram, A.P, India. \\ ${ }^{2}$ Rtd professor in Nagarjuna university,Guntur District,A.P,India. \\ Email: irbsarma44@gmail.com
}

\begin{abstract}
In this paper we establish a common fixed point theorem for two pairs of weakly Compatible maps in dislocated metric( $d$-metric) spaces. The purpose of this note is to prove the $d$-metric versions for weakly compatible maps of new version for Hardy and Rogers[7] theorem which ultimately implies existence (and uniqueness in some cases) of common fixed points for weakly compatible maps that satisfy conditions analogous to those of Banach, Kannan and Chatterjea [5].
\end{abstract}

Keywords: Dislocated metric, weakly compatible maps, Common fixed point, $\alpha$-property.

Mathematics subject classification: $47 \mathrm{H} 10,54 \mathrm{H} 25$

\section{Introduction}

The notion of a dislocated metric space was introduced by Pascal hitzler in [1] as a part of the study of logic programming semantics. The study of common fixed point mappings in dislocated metric space satisfying certain contractive conditions has been at the centre of vigorous research activity.

In 1996, Jungck [4] to the concept of weak compatibility. Since then, many interesting fixed point theorems of compatible and weakly compatible maps under various contractive conditions have been obtained by a number of authors. Hardy and Rogers proved fixed point theorem for a single map in a metric space. In 1922, Banach proved a fixed point theorem for contraction mappings in metric space. 
Since then a number of fixed point theorems have been proved by different authors and many generalization of this theorem have been established. we prove a common fixed point theorem for four maps weakly compatible for Hardy and Rogers[7] type mapping in dislocated metric spaces.

\section{Preliminaries}

Now we start with the following definitions, lemmas and theorems.

Definition $2.1:[3]$ Let $\mathrm{X}$ be a non empty set and let $\mathrm{d}: \mathrm{X} \times \mathrm{X} \rightarrow[0, \infty)$

be a function satisfying the following conditions:

i. $\quad d(x, y)=d(y, x)$

ii. $\quad \mathrm{d}(\mathrm{x}, \mathrm{y})=\mathrm{d}(\mathrm{y}, \mathrm{x})=0$ implies $\mathrm{x}=\mathrm{y}$

iii. $\mathrm{d}(\mathrm{x}, \mathrm{y}) \leq \mathrm{d}(\mathrm{x}, \mathrm{z})+\mathrm{d}(\mathrm{z}, \mathrm{y})$ for all $\mathrm{x}, \mathrm{y}, \mathrm{z} \in \mathrm{X}$.

Then $\mathrm{d}$ is called dislocated metric(or simply $\mathrm{d}$-metric) on $\mathrm{X}$.

Definition 2.2 : [3]A sequence $\left\{\mathrm{x}_{\mathrm{n}}\right\}$ in a $\mathrm{d}$-metric space $(\mathrm{X}, \mathrm{d})$ is called a

Cauchy sequence if given $\in>0$, there corresponds $\mathrm{n}_{0} \in \mathrm{N}$ such that for all $\mathrm{m}, \mathrm{n} \geq \mathrm{n}_{0}$ we have $\mathrm{d}\left(\mathrm{x}_{\mathrm{m}}, \mathrm{x}_{\mathrm{n}}\right)<\epsilon$.

Definition 2.3 : [3] A sequence $\left\{\mathrm{x}_{\mathrm{n}}\right\}$ in $\mathrm{d}$-metric space converges with respect to ' $\mathrm{d}$ ' if there exists $\mathrm{x} \in \mathrm{X}$ such that $\mathrm{d}\left(\mathrm{x}_{\mathrm{n}}, \mathrm{x}\right) \rightarrow 0$ as $\mathrm{n} \rightarrow \infty$.

In this case, $x$ is called limit of $\left\{x_{n}\right\}$ and we write $x_{n} \rightarrow x$.

Definition 2.4 : [3]A d -metric space $(\mathrm{X}, \mathrm{d})$ is called complete if every Cauchy Sequence in it is convergent with respect to $\mathrm{d}$.

Definition 2.5: [3] Let $(\mathrm{X}, \mathrm{d})$ be a $\mathrm{d}$-metric space. A map $\mathrm{T}: \mathrm{X} \rightarrow \mathrm{X}$ is

Called contraction if there exists a number $\lambda$ with $0 \leq \lambda<1$ such that $\mathrm{d}(\mathrm{Tx}, \mathrm{Ty}) \leq \lambda \mathrm{d}(\mathrm{x}, \mathrm{y})$.

We state the following lemmas without proof.

Lemma 2.1: [3]Let $(\mathrm{X}, \mathrm{d})$ be a $\mathrm{d}$-metric space. If $\mathrm{T}: \mathrm{X} \rightarrow \mathrm{X}$ is a contraction function, then $\left\{\mathrm{T}^{\mathrm{n}}\left(\mathrm{x}_{0}\right)\right\}$ is a Cauchy sequence for each $\mathrm{x}_{0} \in \mathrm{X}$.

Lemma 2.7 Limits in a $\mathrm{d}$-metric space are unique.

Definition 2.8: [2] Let $\mathrm{A}$ and $\mathrm{S}$ be mappings from a metric space $(\mathrm{X}, \mathrm{d})$ into itself. Then, $\mathrm{A}$ and $\mathrm{S}$ are said to be weakly compatible if they commute at 
their "coincident point" that is $\mathrm{Ax}=\mathrm{Sx}$ implies $\mathrm{ASx}=\mathrm{SAx}$.

In what follows, $\mathrm{R}^{+}$the set of all positive real numbers.

Definition 2.9:[6]Let a binary operation $\diamond: \mathrm{R}^{+} \times \mathrm{R}^{+} \rightarrow \mathrm{R}^{+}$satisfy the following conditions:

(I) $\diamond$ is Associative and Commutative,

(II) $\diamond$ is continuous w.r.t to the usual metric $\mathrm{R}^{+}$

Definition 2.10:[6]A binary operation $\diamond$ on $\mathrm{R}^{+}$is said to satisfy $\alpha$-property if there exists a positive real number $\alpha$ such that $a \diamond b \leq \alpha \max \{a, b\}$ for every $a, b \in R^{+}$.

A few typical examples are $\mathrm{a} \diamond \mathrm{b}=\max \{\mathrm{a}, \mathrm{b}\}, \mathrm{a} \diamond \mathrm{b}=\mathrm{a}+\mathrm{b}$,

$\mathrm{a} \diamond \mathrm{b}=\mathrm{a} b, \mathrm{a} \diamond \mathrm{b}=\mathrm{a} \mathrm{b}+\mathrm{a}+\mathrm{b}$ and $\mathrm{a} \diamond \mathrm{b}=\frac{\mathrm{ab}}{\max \{\mathrm{a}, \mathrm{b}, 1\}}$

for each $\mathrm{a}, \mathrm{b} \in \mathrm{R}^{+}$. In what follows we fix a binary operation $\diamond$ that satisfies (1) and (11)

Theorem 2.11: [3] Let (X, d) be a complete $\mathrm{d}$-metric space and let $\mathrm{T}: \mathrm{X} \rightarrow \mathrm{X}$ be a contraction mapping, then $\mathrm{T}$ has a unique fixed point.

\section{Main Results}

The following theorem is generalization of theorem 2.4 established by I.Rama Badra Sarma and P Sumati kumari[3].

Theorem 3.1: Let $(\mathrm{X}, \mathrm{d})$ be a complete $\mathrm{d}$-metric space. Let $\mathrm{A}, \mathrm{B}, \mathrm{S}, \mathrm{T}: \mathrm{X} \rightarrow \mathrm{X}$ be continuous mappings satisfying,

(1). $\mathrm{T}(\mathrm{X}) \subset \mathrm{A}(\mathrm{X}), \mathrm{S}(\mathrm{X}) \subset \mathrm{B}(\mathrm{X})$

(2). The pairs $(\mathrm{S}, \mathrm{A})$ and $(\mathrm{T}, \mathrm{B})$ are weakly compatible and

(3). $d(S x, T y) \leq a_{1} d(A x, B y)+a_{2} d(A x, S x)+a_{3} d(B y, T y)+a_{4} d(A x, T y)+a_{5} d(B y, S x)$

For all $\mathrm{x}, \mathrm{y} \in \mathrm{X}$, where $\mathrm{a}_{1}, \mathrm{a}_{2}, \mathrm{a}_{3}, \mathrm{a}_{4}, \mathrm{a}_{5} \geq 0$,

$0 \leq \mathrm{a}_{1}+\mathrm{a}_{2}+\mathrm{a}_{3}+\mathrm{a}_{4}+\mathrm{a}_{5}<\frac{1}{2}$ then $\mathrm{A}, \mathrm{B}, \mathrm{S}$ and $\mathrm{T}$ have a unique common fixed point. 
Proof : Using condition (1), we define sequences $\left\{x_{n}\right\}$ and $\left\{y_{n}\right\}$ in $X$ by the rule,

$\mathrm{y}_{2 \mathrm{n}}=\mathrm{Bx}_{2 \mathrm{n}+1}=\mathrm{Sx}_{2 \mathrm{n}}$ and

$\mathrm{y}_{2 \mathrm{n}+1}=\mathrm{Ax}_{2 \mathrm{n}+2}=\mathrm{Tx}_{2 \mathrm{n}+1}, \mathrm{n}=0,1,2 \ldots$ where $\mathrm{x}_{0}=\mathrm{x}, \mathrm{y}_{0}=\mathrm{y}$

If $\mathrm{y}_{2 \mathrm{n}}=\mathrm{y}_{2 \mathrm{n}+1}$ for some $\mathrm{n}$, then $\mathrm{Bx}_{2 \mathrm{n}+1}=\mathrm{Tx}_{2 \mathrm{n}+1}$. Therefore $\mathrm{x}_{2 \mathrm{n}+1}$

is a coincident point of $B$ and $T$. Also, if $y_{2 n+1}=y_{2 n+2}$ for some $n$, then $A x_{2 n+2}=S x_{2 n+2}$. Hence $x_{2 n+2}$ is a coincident point of $S$ and $A$.

Assume that $\mathrm{y}_{2 \mathrm{n}} \neq \mathrm{y}_{2 \mathrm{n}+1}$ for all $\mathrm{n}$. Then, we have

$$
\begin{aligned}
& d\left(y_{2 n}, y_{2 n+1}\right)=d\left(S_{2 n}, T x_{2 n+1}\right) \\
& \leq a_{1} d\left(A x_{2 n}, B x_{2 n+1}\right)+a_{2} d\left(A x_{2 n}, S x_{2 n}\right)+a_{3} d\left(B x_{2 n+1}, T_{2 n+1}\right) \\
& +a_{4} d\left(A x_{2 n}, T x_{2 n+1}\right)+a_{5} d\left(B x_{2 n+1}, S x_{2 n}\right) \\
& \leq a_{1} d\left(y_{2 n-1}, y_{2 n}\right)+a_{2} d\left(y_{2 n-1}, y_{2 n}\right)+a_{3} d\left(y_{2 n}, y_{2 n+1}\right) \leq\left(a_{1}+a_{2}+a_{4}\right) d\left(y_{2 n-1}, y_{2 n}\right) \\
& +a_{4} d\left(y_{2 n-1}, y_{2 n+1}\right)+a_{5} d\left(y_{2 n}, y_{2 n}\right) \\
& \left(1-a_{3}-a_{4}-2 a_{5}\right) d\left(y_{2 n}, y_{2 n+1}\right) \leq\left(a_{1}+a_{2}+a_{4}\right) d\left(y_{2 n-1}, y_{2 n}\right) \\
& d\left(y_{2 n}, y_{2 n+1}\right) \leq\left(\frac{a_{1}+a_{2}+a_{4}}{1-a_{3}-a_{4}-2 a_{5}}\right) d\left(y_{2 n-1}, y_{2 n}\right) \\
& \text { where h }=\left(\frac{a_{1}+a_{2}+a_{4}}{1-a_{3}-a_{4}-2 a_{5}}\right)<1
\end{aligned}
$$

This shows that

$d\left(y_{2 n}, y_{2 n+1}\right) \leq h d\left(y_{n-1}, y_{n}\right) \leq \ldots \leq h^{n} d\left(y_{0}, y_{n}\right)$

For every integer $\mathrm{q}>0$ we have 


$$
\begin{aligned}
& d\left(y_{n}, y_{n+q}\right) \leq d\left(y_{n}, y_{n+1}\right)+d\left(y_{n+1}, y_{n+2}\right)+d\left(y_{n+2}, y_{n+3}\right)+\ldots+d\left(y_{n+q-1}, y_{n+q}\right) \\
& \quad \leq\left(1+h+h^{2}+\ldots+h^{q-1}\right) d\left(y_{n}, y_{n+1}\right) \\
& \leq \frac{h^{n}}{1-h} d\left(y_{0}, y_{1}\right) \\
& \quad \text { Since, } 0<h<1, h^{n} \rightarrow 0 \text { as } n \rightarrow \infty
\end{aligned}
$$

So, we get $d\left(y_{n}, y_{n+q}\right) \rightarrow 0$ this implies $\left\{y_{n}\right\}$ is a Cauchy sequence in a Complete dislocated metric space. So, there exists a point $\mathrm{z} \in \mathrm{X}$ such that $\left\{\mathrm{y}_{\mathrm{n}}\right\} \rightarrow \mathrm{z}$.

Therefore the subsequences,

$\left\{\mathrm{Sx}_{2 \mathrm{n}}\right\} \rightarrow \mathrm{z}, \quad\left\{\mathrm{Bx}_{2 \mathrm{n}+1}\right\} \rightarrow \mathrm{z}, \quad\left\{\mathrm{Tx}_{2 \mathrm{n}+1}\right\} \rightarrow \mathrm{z}$ and $\left\{\mathrm{Ax}_{2 \mathrm{n}+2}\right\} \rightarrow \mathrm{z}$

Since $\mathrm{T}(\mathrm{X}) \subset \mathrm{A}(\mathrm{X})$, there exists a point $\mathrm{u} \in \mathrm{X}$ such that $\mathrm{z}=\mathrm{Au}$.

So, $\quad d(S u, z)=d\left(S u, \mathrm{Tx}_{2 \mathrm{n}+1}\right)$

$$
\begin{gathered}
\leq \mathrm{a}_{1} \mathrm{~d}\left(\mathrm{Au}, \mathrm{Bx}_{2 \mathrm{n}+1}\right)+\mathrm{a}_{2} \mathrm{~d}(\mathrm{Au}, \mathrm{Su})+\mathrm{a}_{3} \mathrm{~d}\left(\mathrm{Bx}_{2 \mathrm{n}+1}, \mathrm{Tx}_{2 \mathrm{n}+1}\right)+\mathrm{a}_{4} \mathrm{~d}\left(\mathrm{Au}, \mathrm{Tx}_{2 \mathrm{n}+1}\right) \\
+\mathrm{a}_{5} \mathrm{~d}\left(\mathrm{Bx}_{2 \mathrm{n}+1}, \mathrm{Su}\right) \\
\leq \mathrm{a}_{1} \mathrm{~d}\left(\mathrm{z}, \mathrm{Bx}_{2 \mathrm{n}+1}\right)+\mathrm{a}_{2} \mathrm{~d}(\mathrm{z}, \mathrm{Su})+\mathrm{a}_{3} \mathrm{~d}\left(\mathrm{Bx}_{2 \mathrm{n}+1}, \mathrm{Tx}_{2 \mathrm{n}+1}\right)+\mathrm{a}_{4} \mathrm{~d}\left(\mathrm{z}, \mathrm{Tx}_{2 \mathrm{n}+1}\right) \\
+\mathrm{a}_{5} \mathrm{~d}\left(\mathrm{Bx}_{2 \mathrm{n}+1}, \mathrm{Su}\right)
\end{gathered}
$$

Taking limits as $\mathrm{n} \rightarrow \infty$ we get,

$$
\begin{aligned}
\mathrm{d}(\mathrm{Su}, \mathrm{z}) & \leq \mathrm{a}_{1} \mathrm{~d}(\mathrm{z}, \mathrm{z})+\mathrm{a}_{2} \mathrm{~d}(\mathrm{z}, \mathrm{Su})+\mathrm{a}_{3} \mathrm{~d}(\mathrm{z}, \mathrm{z})+\mathrm{a}_{4} \mathrm{~d}(\mathrm{z}, \mathrm{z})+\mathrm{a}_{5} \mathrm{~d}(\mathrm{z}, \mathrm{Su}) \\
& \leq\left(\mathrm{a}_{1}+\mathrm{a}_{3}+\mathrm{a}_{4}\right) \mathrm{d}(\mathrm{z}, \mathrm{z})+\left(\mathrm{a}_{2}+\mathrm{a}_{5}\right) \mathrm{d}(\mathrm{z}, \mathrm{Su}) \\
& \leq\left[2\left(\mathrm{a}_{1}+\mathrm{a}_{3}+\mathrm{a}_{4}\right)+\left(\mathrm{a}_{2}+\mathrm{a}_{5}\right)\right] \mathrm{d}(\mathrm{z}, \mathrm{Su})
\end{aligned}
$$

Which is a contradiction since $\left[2\left(a_{1}+a_{3}+a_{4}\right)+\left(a_{2}+a_{5}\right)\right]<1$

So we have $\mathrm{Su}=\mathrm{Au}=\mathrm{z}$

Again since $S(X) \subset B(X)$ there exists a point $v \in X$ such that $z=B v$

We claim that $\mathrm{z}=\mathrm{T} \mathrm{v}$ 


$$
\begin{aligned}
& \mathrm{d}(\mathrm{z}, \mathrm{Tv})=\mathrm{d}(\mathrm{Su}, \mathrm{Tv}) \\
& \leq \mathrm{a}_{1} \mathrm{~d}(\mathrm{Au}, \mathrm{Bv})+\mathrm{a}_{2} \mathrm{~d}(\mathrm{Au}, \mathrm{Su})+\mathrm{a}_{3} \mathrm{~d}(\mathrm{Bv}, \mathrm{Tv})+\mathrm{a}_{4} \mathrm{~d}(\mathrm{Au}, \mathrm{Tv})+\mathrm{a}_{5} \mathrm{~d}(\mathrm{Bv}, \mathrm{Su}) \\
& \leq \mathrm{a}_{1} \mathrm{~d}(\mathrm{z}, \mathrm{z})+\mathrm{a}_{2} \mathrm{~d}(\mathrm{z}, \mathrm{z})+\mathrm{a}_{3} \mathrm{~d}(\mathrm{z}, \mathrm{Tv})+\mathrm{a}_{4} \mathrm{~d}(\mathrm{z}, \mathrm{Tv})+\mathrm{a}_{5} \mathrm{~d}(\mathrm{z}, \mathrm{z}) \\
& \leq\left(\mathrm{a}_{1}+\mathrm{a}_{2}+\mathrm{a}_{5}\right) \mathrm{d}(\mathrm{z}, \mathrm{z})+\left(\mathrm{a}_{3}+\mathrm{a}_{4}\right) \mathrm{d}(\mathrm{z}, \mathrm{Tv}) \\
& \leq\left[2\left(\mathrm{a}_{1}+\mathrm{a}_{2}+\mathrm{a}_{5}\right)+\left(\mathrm{a}_{3}+\mathrm{a}_{4}\right)\right] \mathrm{d}(\mathrm{z}, \mathrm{Tv}) \\
& \Rightarrow \mathrm{d}(\mathrm{z}, \mathrm{Tv})=0 \quad \text { since }\left[2\left(\mathrm{a}_{1}+\mathrm{a}_{2}+\mathrm{a}_{5}\right)+\left(\mathrm{a}_{3}+\mathrm{a}_{4}\right)\right]<1
\end{aligned}
$$

So we get $\mathrm{z}=\mathrm{Tv}$

Hence $\mathrm{Su}=\mathrm{Au}=\mathrm{Tv}=\mathrm{Bv}=\mathrm{z}$.

Since the pair $(\mathrm{S}, \mathrm{A})$ is weakly compatible, by definition $\mathrm{SAu}=\mathrm{ASu}$ implies $\mathrm{Sz}=\mathrm{Az}$.

Now, we show that $\mathrm{z}$ is the fixed point of $\mathrm{S}$.

$$
\begin{aligned}
& \mathrm{d}(\mathrm{Sz}, \mathrm{z})=\mathrm{d}(\mathrm{Sz}, \mathrm{Tz}) \\
& \leq \mathrm{a}_{1} \mathrm{~d}(\mathrm{Az}, \mathrm{Bv})+\mathrm{a}_{2} \mathrm{~d}(\mathrm{Az}, \mathrm{Sz})+\mathrm{a}_{3} \mathrm{~d}(\mathrm{Bv}, \mathrm{Tv})+\mathrm{a}_{4} \mathrm{~d}(\mathrm{Az}, \mathrm{Tv})+\mathrm{a}_{5} \mathrm{~d}(\mathrm{Bv}, \mathrm{Sz}) \\
& \leq \mathrm{a}_{1} \mathrm{~d}(\mathrm{Sz}, \mathrm{z})+\mathrm{a}_{2} \mathrm{~d}(\mathrm{Sz}, \mathrm{Sz})+\mathrm{a}_{3} \mathrm{~d}(\mathrm{z}, \mathrm{z})+\mathrm{a}_{4} \mathrm{~d}(\mathrm{Sz}, \mathrm{z})+\mathrm{a}_{5} \mathrm{~d}(\mathrm{z}, \mathrm{Sz}) \\
& \leq\left(\mathrm{a}_{1}+2 \mathrm{a}_{2}+2 \mathrm{a}_{3}+\mathrm{a}_{4}+\mathrm{a}_{5}\right) \mathrm{d}(\mathrm{z}, \mathrm{Sz}) \text { which is a contradiction. } \\
& \because \mathrm{a}_{1}+2 \mathrm{a}_{2}+2 \mathrm{a}_{3}+\mathrm{a}_{4}+\mathrm{a}_{5}<1
\end{aligned}
$$

so we have $\mathrm{Sz}=\mathrm{z}=\mathrm{Az}$.

Again, the pair $(\mathrm{T}, \mathrm{B})$ are weakly compatible, so by definition $\mathrm{TBv}=\mathrm{BTV}$ implies $\mathrm{Tz}=\mathrm{Bz}$.

Now, we show that ' $z$ ' is the fixed point of $T$.

$$
\begin{aligned}
& \mathrm{d}(\mathrm{z}, \mathrm{Tz})=\mathrm{d}(\mathrm{Sz}, \mathrm{Tz}) \\
& \quad \leq \mathrm{a}_{1} \mathrm{~d}(\mathrm{Az}, \mathrm{Bz})+\mathrm{a}_{2} \mathrm{~d}(\mathrm{Az}, \mathrm{Sz})+\mathrm{a}_{3} \mathrm{~d}(\mathrm{Bz}, \mathrm{Tz})+\mathrm{a}_{4} \mathrm{~d}(\mathrm{Az}, \mathrm{Tz})+\mathrm{a}_{5} \mathrm{~d}(\mathrm{Bz}, \mathrm{Sz}) \\
& \quad \leq \mathrm{a}_{1} \mathrm{~d}(\mathrm{z}, \mathrm{Tz})+\mathrm{a}_{2} \mathrm{~d}(\mathrm{z}, \mathrm{z})+\mathrm{a}_{3} \mathrm{~d}(\mathrm{Tz}, \mathrm{Tz})+\mathrm{a}_{4} \mathrm{~d}(\mathrm{z}, \mathrm{Tz})+\mathrm{a}_{5} \mathrm{~d}(\mathrm{Tz}, \mathrm{z}) \\
& \quad \leq\left(\mathrm{a}_{1}+2 \mathrm{a}_{2}+2 \mathrm{a}_{3}+\mathrm{a}_{4}+\mathrm{a}_{5}\right) \mathrm{d}(\mathrm{z}, \mathrm{Tz}) \text { which is a contradiction. } \\
& \because \mathrm{a}_{1}+2 \mathrm{a}_{2}+2 \mathrm{a}_{3}+\mathrm{a}_{4}+\mathrm{a}_{5}<1 \\
& \therefore \mathrm{Tz}=\mathrm{z}
\end{aligned}
$$

Hence we have $\mathrm{Az}=\mathrm{Bz}=\mathrm{Sz}=\mathrm{Tz}=\mathrm{z}$. This shows that ' $\mathrm{z}$ ' is the common fixed point of the self mappings A, B,S and T. 
Uniqueness: Let $\mathrm{u} \neq \mathrm{v}$ be two common fixed points of the mappings $\mathrm{A}, \mathrm{B}, \mathrm{S}$ and T.

Then we have,

$$
\begin{aligned}
& \mathrm{d}(\mathrm{u}, \mathrm{v})= \\
& \quad \mathrm{d}(\mathrm{Su}, \mathrm{Tv}) \\
& \quad \leq \mathrm{a}_{1} \mathrm{~d}(\mathrm{Au}, \mathrm{Bv})+\mathrm{a}_{2} \mathrm{~d}(\mathrm{Au}, \mathrm{Su})+\mathrm{a}_{3} \mathrm{~d}(\mathrm{Bv}, \mathrm{Tv})+\mathrm{a}_{4} \mathrm{~d}(\mathrm{Au}, \mathrm{Tv})+\mathrm{a}_{5} \mathrm{~d}(\mathrm{Bv}, \mathrm{Su}) \\
& \quad \leq \mathrm{a}_{1} \mathrm{~d}(\mathrm{u}, \mathrm{v})+\mathrm{a}_{2} \mathrm{~d}(\mathrm{u}, \mathrm{u})+\mathrm{a}_{3} \mathrm{~d}(\mathrm{v}, \mathrm{v})+\mathrm{a}_{4} \mathrm{~d}(\mathrm{u}, \mathrm{v})+\mathrm{a}_{5} \mathrm{~d}(\mathrm{v}, \mathrm{u}) \\
& \quad \leq\left(\mathrm{a}_{1}+2 \mathrm{a}_{2}+2 \mathrm{a}_{3}+\mathrm{a}_{4}+\mathrm{a}_{5}\right) \mathrm{d}(\mathrm{u}, \mathrm{v}) \text { which is a contradiction. } \\
& \because \mathrm{a}_{1}+2 \mathrm{a}_{2}+2 \mathrm{a}_{3}+\mathrm{a}_{4}+\mathrm{a}_{5}<1 \\
& \therefore \mathrm{d}(\mathrm{u}, \mathrm{v})=0
\end{aligned}
$$

Since $(\mathrm{X}, \mathrm{d})$ is a dislocated metric space, so we have $\mathrm{u}=\mathrm{v}$.

Put $\mathrm{A}=\mathrm{B}=\mathrm{I}$ an identity mapping in above theorem 3.1 yields corollary 3.2

Corollary 3.2: Let $(\mathrm{X}, \mathrm{d})$ be a complete $\mathrm{d}$-metric space. Let $\mathrm{S}, \mathrm{T}: \mathrm{X} \rightarrow \mathrm{X}$ be continuous mappings satisfying,

$\mathrm{d}(\mathrm{Sx}, \mathrm{Ty}) \leq \mathrm{a}_{1} \mathrm{~d}(\mathrm{x}, \mathrm{y})+\mathrm{a}_{2} \mathrm{~d}(\mathrm{x}, \mathrm{Sx})+\mathrm{a}_{3} \mathrm{~d}(\mathrm{y}, \mathrm{Ty})+\mathrm{a}_{4} \mathrm{~d}(\mathrm{x}, \mathrm{Ty})+\mathrm{a}_{5} \mathrm{~d}(\mathrm{y}, \mathrm{Sx}) \quad$ for $\quad$ all $\mathrm{x}, \mathrm{y} \in \mathrm{X}$, where $\mathrm{a}_{1}+\mathrm{a}_{2}+\mathrm{a}_{3}+\mathrm{a}_{4}+\mathrm{a}_{5}<\frac{1}{2}$ then $\mathrm{S}$ and $\mathrm{Thave}$ unique fixed point.

If $\mathrm{S}=\mathrm{T}$ in corollary 3.2 yields corollary 3.3

Corollary 3.2: Let $(\mathrm{X}, \mathrm{d})$ be a complete $\mathrm{d}$-metric space. Let $\mathrm{T}: \mathrm{X} \rightarrow \mathrm{X}$ be a continuous mapping satisfying,

$\mathrm{d}(\mathrm{Tx}, \mathrm{Ty}) \leq \mathrm{a}_{1} \mathrm{~d}(\mathrm{x}, \mathrm{y})+\mathrm{a}_{2} \mathrm{~d}(\mathrm{x}, \mathrm{Tx})+\mathrm{a}_{3} \mathrm{~d}(\mathrm{y}, \mathrm{Ty})+\mathrm{a}_{4} \mathrm{~d}(\mathrm{x}, \mathrm{Ty})+\mathrm{a}_{5} \mathrm{~d}(\mathrm{y}, \mathrm{Tx}) \quad$ for $\quad$ all $\mathrm{x}, \mathrm{y} \in \mathrm{X}$, where $\mathrm{a}_{1}+\mathrm{a}_{2}+\mathrm{a}_{3}+\mathrm{a}_{4}+\mathrm{a}_{5}<\frac{1}{2}$ then $\mathrm{T}$ has a unique fixed point.

If we take $\mathrm{A}=\mathrm{T}$ and $\mathrm{B}=\mathrm{S}$ in the theorem 3.1 yields corollary 3.4

Corollary 3.3: Let $(\mathrm{X}, \mathrm{d})$ be a complete $\mathrm{d}$-metric space. Let $\mathrm{S}, \mathrm{T}: \mathrm{X} \rightarrow \mathrm{X}$ be continuous mappings satisfying,

$d(S x, T y) \leq a_{1} d(T x, S y)+a_{2} d(T x, S x)+a_{3} d(S y, T y)+a_{4} d(T x, T y)+a_{5} d(S y, S x)$ for all $\mathrm{x}, \mathrm{y} \in \mathrm{X}$.

where $\mathrm{a}_{1}, \mathrm{a}_{2}, \mathrm{a}_{3}, \mathrm{a}_{4}, \mathrm{a}_{5} \geq 0, \mathrm{a}_{1}+\mathrm{a}_{2}+\mathrm{a}_{3}+\mathrm{a}_{4}+\mathrm{a}_{5}<\frac{1}{2}$.

then Sand Thave unique fixed point. 
B.E Rhodes [5] presented a list of definitions of contractive type conditions for a self map on a metric space ( $\mathrm{X}, \mathrm{d})$ and established implications and non implications among them ,there by facilitating to check the implication of any new contractive condition any one of the condition mentioned in [5] so as to derive a fixed point theorem. Among the conditions in [5], Hardy and Rogers condition is significant as a good number of Contractive conditions imply Hardy and Rogers's condition. We now present the dislocated versions of these conditions.

Let $(X, d)$ be a dislocated metric space and $A, B, S, T: X \rightarrow X$ be a mappings and $\mathrm{x}, \mathrm{y}$ be any elements of $\mathrm{X}$.

Consider the following conditions,

1. (Banach) :there exists a number $\alpha, 0 \leq \alpha \leq 1$ such that for each

$$
\begin{aligned}
& \mathrm{x}, \mathrm{y} \quad \in \mathrm{X} \\
& \mathrm{d}(\mathrm{Sx}, \mathrm{Ty}) \leq \alpha \mathrm{d}(\mathrm{Ax}, \mathrm{By}) .
\end{aligned}
$$

2. (Kannan) :there exists a number $\alpha, 0<\alpha<\frac{1}{2}$ such that $\mathrm{d}(\mathrm{Sx}, \mathrm{Ty})<\alpha[\mathrm{d}(\mathrm{Ax}, \mathrm{Sx})+\mathrm{d}(\mathrm{By}, \mathrm{Ty})]$

3. (Chatterjea): There exists a number a, $0<\mathrm{a}<\frac{1}{2}$ such that, for each $\mathrm{x}, \mathrm{y} \in \mathrm{X}$,

$$
\mathrm{d}(\mathrm{Sx}, \mathrm{Ty}) \leq \mathrm{a}[\mathrm{d}(\mathrm{Ax}, \mathrm{Ty})+\mathrm{d}(\mathrm{By}, \mathrm{Sx})] .
$$

4. (Hardy and Rogers): There exist non negative constants $\mathrm{a}_{\mathrm{i}}$ satisfying $0 \leq \mathrm{a}_{1}+\mathrm{a}_{2}+\mathrm{a}_{3}+\mathrm{a}_{4}+\mathrm{a}_{5}<\frac{1}{2} \quad$ such that, for each $\mathrm{x}, \mathrm{y} \in \mathrm{X}$, $\mathrm{d}(\mathrm{Sx}, \mathrm{Ty}) \leq \mathrm{a}_{1} \mathrm{~d}(\mathrm{Ax}, \mathrm{By})+\mathrm{a}_{2} \mathrm{~d}(\mathrm{Ax}, \mathrm{Sx})+\mathrm{a}_{3} \mathrm{~d}(\mathrm{By}, \mathrm{Ty})+\mathrm{a}_{4} \mathrm{~d}(\mathrm{Ax}, \mathrm{Ty})+\mathrm{a}_{5} \mathrm{~d}(\mathrm{By}, \mathrm{Sx})$

Theorem 3.4: Let $(\mathrm{X}, \mathrm{d})$ be a complete $\mathrm{d}$-metric space. Let $\mathrm{A}, \mathrm{B}, \mathrm{S}, \mathrm{T}: \mathrm{X} \rightarrow \mathrm{X}$ be continuous mapping satisfying,

I. $\quad \mathrm{T}(\mathrm{X}) \subset \mathrm{A}(\mathrm{X}), \mathrm{S}(\mathrm{X}) \subset \mathrm{B}(\mathrm{X})$

II. The pairs (S, A)and(T,B) are weakly compatible and if $\mathrm{A}, \mathrm{B}, \mathrm{S}, \mathrm{T}: \mathrm{X} \rightarrow \mathrm{X}$ satisfies any one of the conditions (1) through (3) then $\mathrm{A}, \mathrm{B}, \mathrm{S}$ and $\mathrm{T}$ have a unique common fixed points.

Proof : In [5] B.E Rhodes proved that when $d$ is a metric.

$$
\text { (1) } \Rightarrow(4)
$$




$$
\begin{aligned}
& (2) \Rightarrow(4) \\
& (3) \Rightarrow(4)
\end{aligned}
$$

With $\mathrm{d}(\mathrm{x}, \mathrm{y}) \neq 0$ is replaced by $\mathrm{x} \neq \mathrm{y}$.Consequently these implications hold good in a $d$-metric space as well since $x \neq y \Rightarrow d(x, y) \neq 0$ in a $d$-metric space .It now follows from theorem 3.1 that $\mathrm{A}, \mathrm{B}, \mathrm{S}$ and $\mathrm{T}$ have a unique common fixed point.

Corollary 3.5: Let $(X, d)$ be a complete $d$-metric space such that $\diamond$ satisfies $\alpha$ property with $\alpha<1$. Let $\mathrm{A}, \mathrm{B}, \mathrm{S}, \mathrm{T}: \mathrm{X} \rightarrow \mathrm{X}$ be continuous mapping satisfying,

i. $\quad \mathrm{T}(\mathrm{X}) \subset \mathrm{A}(\mathrm{X}), \mathrm{S}(\mathrm{X}) \subset \mathrm{B}(\mathrm{X})$

ii. The pairs $(\mathrm{S}, \mathrm{A})$ and $(\mathrm{T}, \mathrm{B})$ are weakly compatible and

iii. $\quad d(S x, T y) \leq a_{1} d(A x, B y) \diamond a_{2} d(A x, S x) \diamond a_{3} d(B y, T y) \diamond a_{4} d(A x, T y) \diamond a_{5} d(B y, S x)$

For all $\mathrm{x}, \mathrm{y} \in \mathrm{X}$, where $\mathrm{a}_{1}, \mathrm{a}_{2}, \mathrm{a}_{3}, \mathrm{a}_{4}, \mathrm{a}_{5} \geq 0$,

$0 \leq \mathrm{a}_{1}+\mathrm{a}_{2}+\mathrm{a}_{3}+\mathrm{a}_{4}+\mathrm{a}_{5}<\frac{1}{2}$ then $\mathrm{A}, \mathrm{B}, \mathrm{S}$ and $\mathrm{T}$ have a unique common fixed points.

Proof: Define $a \diamond b=a+b$ for all $a, b \in R^{+}$.Then we have $a \diamond b=\alpha \max \{a, b\}$ and hence all conditions of theorem 3.1 holds. Thus A, B,S and Thave a unique common fixed points.

\section{References:}

[1] P. Hitzler, Generalized Metrics and Topology in Logic Programming Semantics, Ph.D.Thesis, National University of Ireland,(University College, Cork), 2001.

[2] Gerald jungck, Proceedings of the American Mathematical Society, vol. 103, no.3July $1988,977-983$

[3] P Sumati kumari, On dislocated quasi metrics, Jour. of Adv. Stu. in Top,Vol. 3, no. 2,2012, $66-74$.

[4] G. Jungck, Common fixed points for noncontinuous nonself mappings on a nonmetricspace, Far East J. Math. Sci. 4(2)(1996), 199-212.

[5] B.E.Rhoades, A comparison of various definitions of contractive mappings,Trans of the Amer.Math.Society vol 226(1977) 257-290.

[6] S.Sedghi and N.Shobe, Common fixed point theorems for four mappings in complete Metric space, Bulletin of the Iranian Mathematical Society Vol. 33 No. 2 (2007), pp 37-47

[7] G E Hardy and T D Rogers, A Generalization of a fixed point theorem of Reich,canad. Math. Bull. 16, 201-206,(1973). 